
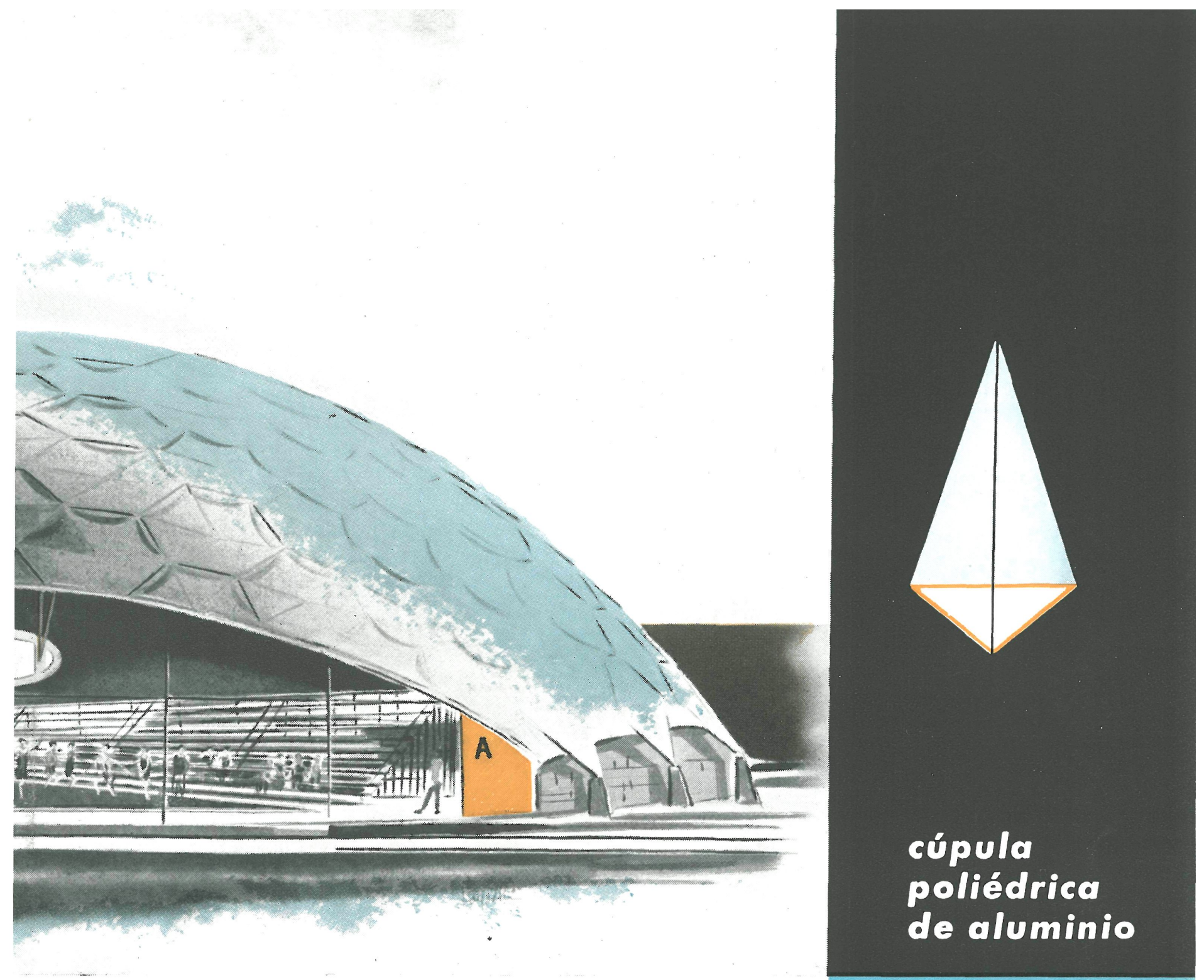

$443-4$

En las islas Hawai se ha construído una gran nave, circular en planta, de 44,20 m de diámetro, destinada a celebrar actos culturales, audiciones, teatro, etcétera, cubierta con una cúpula poliédrica de la clase icosaédrica y formando un segmento de esfera de $24,40 \mathrm{~m}$. de radio.

Su construcción ha sido proyectada y dirigida por Product Development of Kaiser Aluminum \& Chemical Corporation.

Esta cubierta, autorresistente, está constituída por 575 paneles de chapa de aluminio, de unos $2 \mathrm{~mm}$ de espesor, de proyección horizontal romboidal de diez diferentes tipos. Cada uno de estos paneles tiene una forma abarquillada, de eje definido por la diagonal entre vértices opuestos y más distanciados. Normal a este eje, y en un plano superior, se unen los otros dos vértices del panel, por medio de una riostra, también de aluminio, de sección en U. Los vértices salientes del icosaedro están todos inscritos en la esfera, de $24,40 \mathrm{~m}$ de radio, que circunscribe a la cúpula.

El procedimiento empleado para determinar el número de rombos y los diferentes tipos de éstos se ha basado en el método de R. Buckminster Fuller para la subdivisión del icosaedro esférico en un reticulado de tres direcciones. 

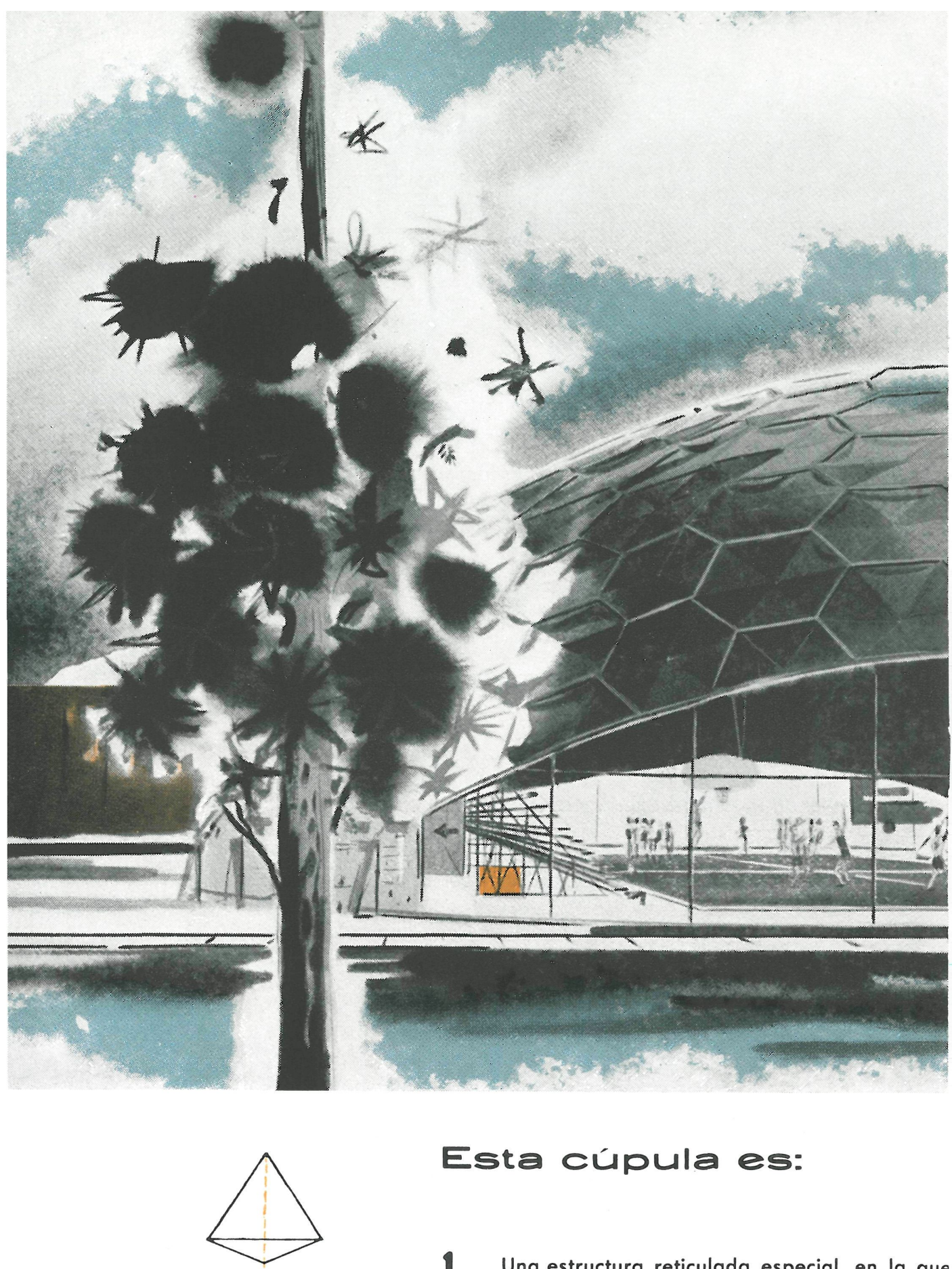

Esta cúpula es:

Una estructura reticulada especial, en la que el tetraedro es su elemento modular.

2 Una estructura autorresistente.

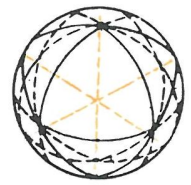

3 Una estructura geodésica, ya que los vértices tetraédricos están circunscritos en una esfera.

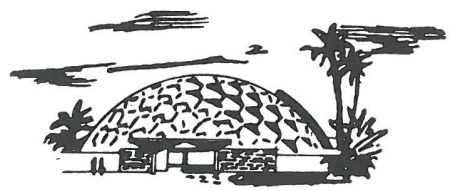

4 Una estructura laminar, en la que la agrupación de tetraedros representa un segmento esférico. 


\section{elemento tipo}

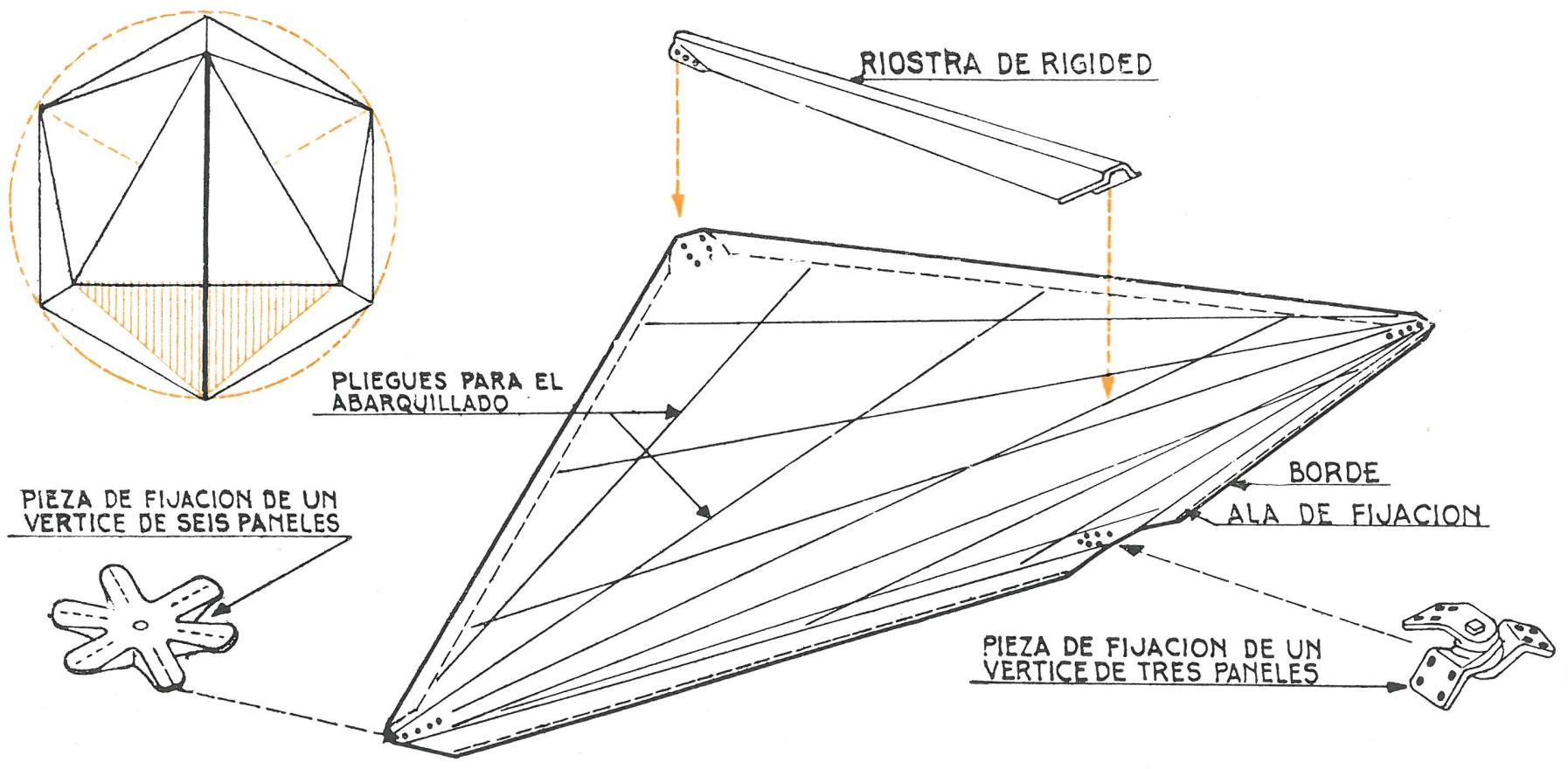

La casa Kaiser Aluminum retiene la exclusiva de la patente que Mr. Fuller ha conseguido sobre las partes constituyentes del icosaedro esférico según su método.

Los cimientos están formados por una corona circular, de hormigón armado, que se apoya sobre un banco de caliza coraloide, descubierta a unos $1,80 \mathrm{~m}$ bajo la superficie. La losa que constituye el solado, de $10 \mathrm{~cm}$ de espesor, junto con la masa de cimientos, forma una sólida estructura capaz de absorber los esfuerzos que pudieran derivarse de la acción de un viento huracanado.

Sobre la corona de cimientos se levantan los 25 soportes que sirven de apoyo a la cúpula poliédrica. Estos soportes tienen una altura, variable, de 0,45 a 1,65 metros.

La cubierta icosaédrica no tiene más elemento resistente que su propia estructura, formada por paneles que se unen entre sí mediante tornillos que solidarizan los bordes.

La unión de seis paneles alrededor de un punto forma una estrella de seis puntas, en cuyo vértice central o lugar de convergencia se solidarizan los seis paneles con una grapa en roseta de seis pétalos, en cada uno de los cuales van cuatro tornillos de fijación, constituyendo así una agrupación parcial de paneles y un tipo de vértices.

Si se unen tres paneles convergiendo los ejes menores en un punto central obtendremos un hexágono, en cuyo vértice central se fijan los paneles por medio de tres grapas con un mismo eje de rotación y provistas, cada una de ellas, de dos filas de dos pernos de fijación.

En esta última forma parcial de unión entre paneles da lugar a la convergencia de las tres riostras que unen los dos vértices del eje menor del panel. Seis nudos de esta base, dispuestos alrededor del punto central de convergencia, da seis paneles que, colocados radialmente siguiendo la dirección de sus ejes mayores, dan lugar a la formación de un hexágono delimitado por seis riostras.

El abarquillamiento de los paneles elementales, cuyos ejes mayores son de 2,69 a 3,55 m y de 1,65 a 2,08 $\mathrm{m}$ los menores, se realizó por medio de una prensa-estampa y partiendo de doce pliegues, seis en cada uno de los lados abarquillados.

Estos abarquillamientos dan lugar a una serie de bajantes, de dirección radial y algo cruzada a esta dirección en otro sentido, que permiten un 
rápido drenaje de aguas y de difícil acumulación de polvo u otras suciedades.

La unión de la cúpula propiamente dicha a sus 25 soportes, dispuestos según una circunferencia, se realiza por el intermedio de una placa anclada sobre cada uno de los soportes. Sobre esta placa, regulable en altura, se apoya una pieza metálica, de sección en U, que se fija con pernos a la placa de base. Dentro de esta última pieza se ajusta un montante, atornillado en la extremidad superior al vértice formado por la convergencia de seis paneles y en su extremidad inferior a la pieza de sección en forma de U. También se apoya en esta pieza la parte inferior del panel que sobresale del resto de la cúpula en cada uno de los soportes.
Entre apoyos, la luz se salva con el arco que forma el borde inferior del último anillo de paneles.

La estructura así formada con estos paneles presenta una magnífica estabilidad y resistencia, prestándose a diferentes formas y modalidades de cierre lateral $o$, si se quiere, dejarla abierta en las proximidades del suelo.

Como interiormente no se apoya en parte alguna, su altura, de $15 \mathrm{~m}$ en este caso particular, puede variar según el diámetro del anillo de apoyo y la altura de los soportes. El aspecto externo es muy agradable, y el interno constituye un atrayente medio de decoración, no sólo por su forma geométrica, sino por su propia novedad actual, a lo que se añade el propio brillo del metal.

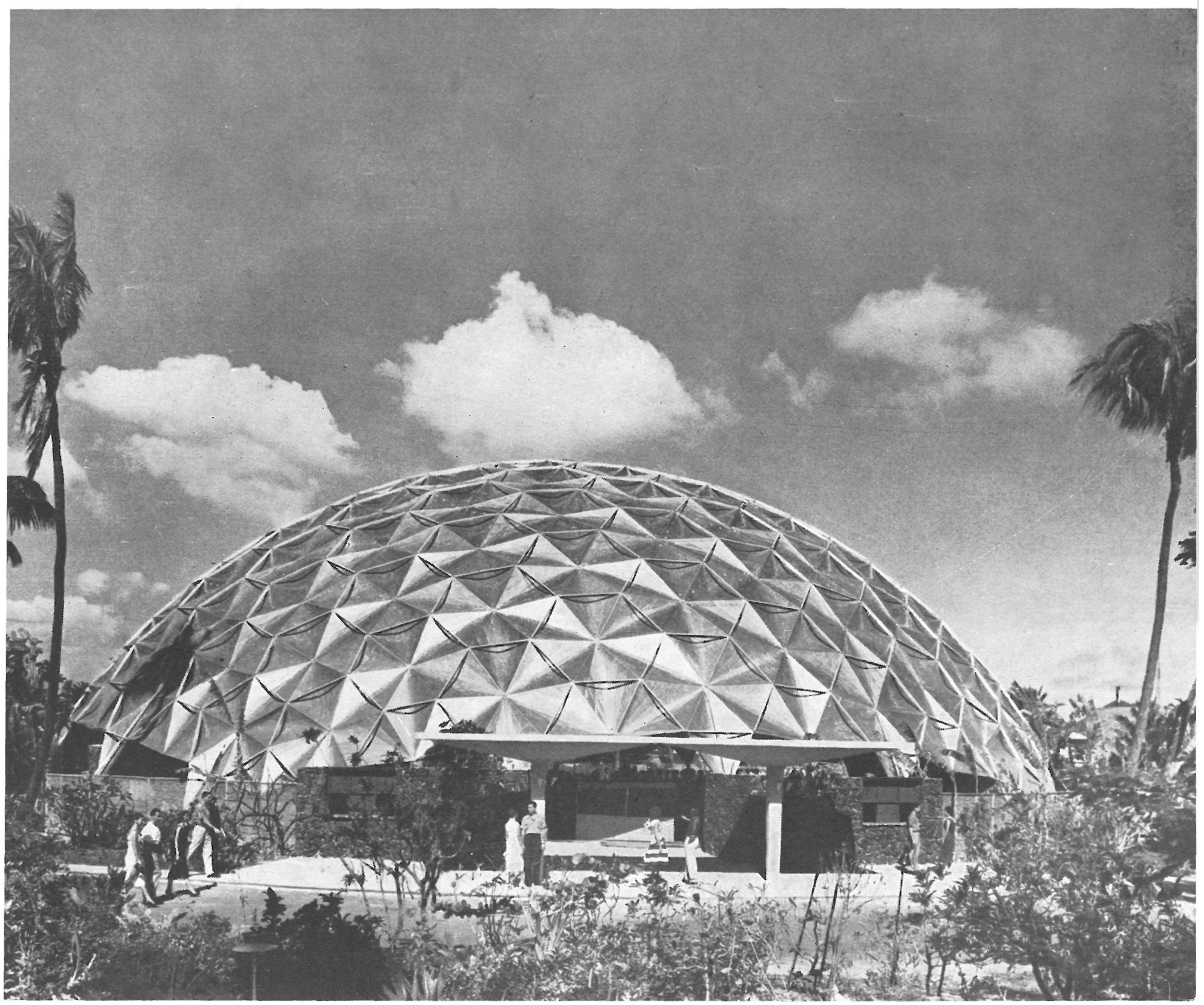




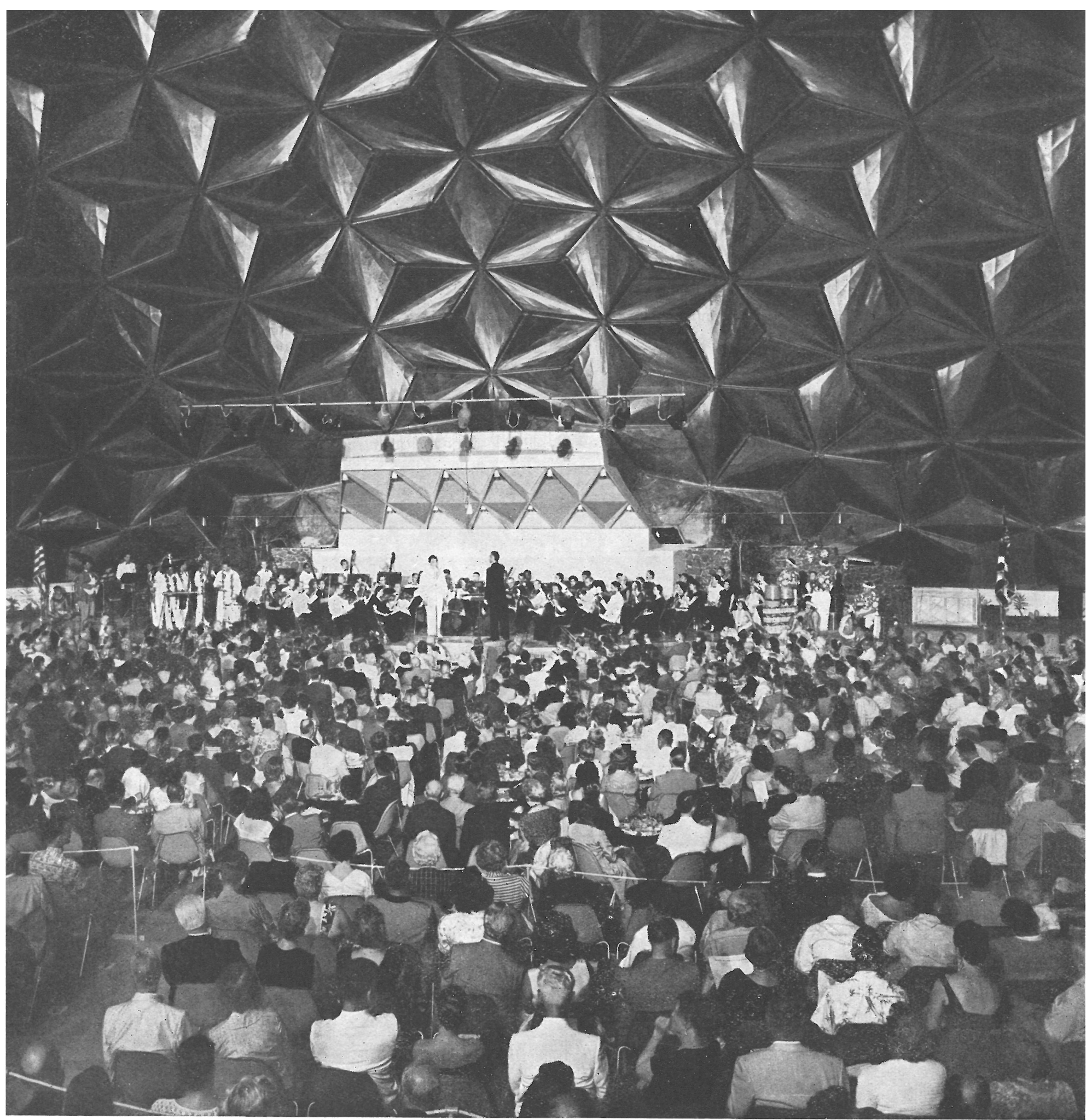

El peso total de la cúpula es de unas 17 toneladas en esta cubierta, y, después de ensayos de carga sobre un modelo natural, la cúpula ha resistido más de 488 kilogramos por metro cuadrado.

Preparados los materiales, se empieza por levantar un castillete central, el cual servirá para ir sosteniendo la cúpula a distintas alturas a medida que avanza su construcción. De lo alto de este castillete parten una serie de vientos, que se fijan en la parte exterior de la cúpula. 


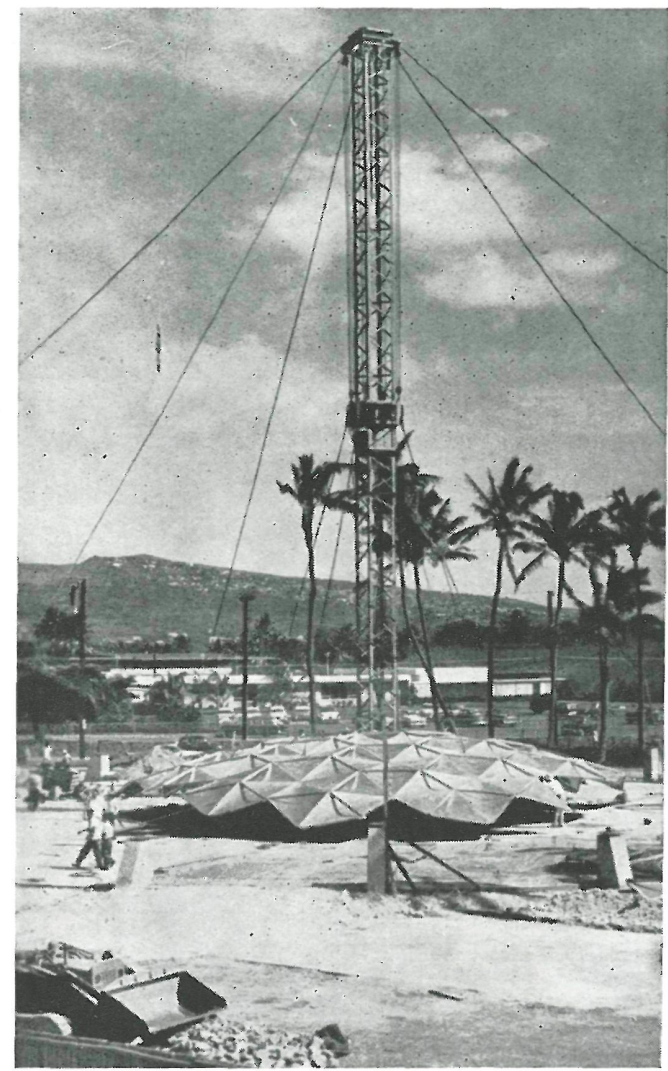

construcción

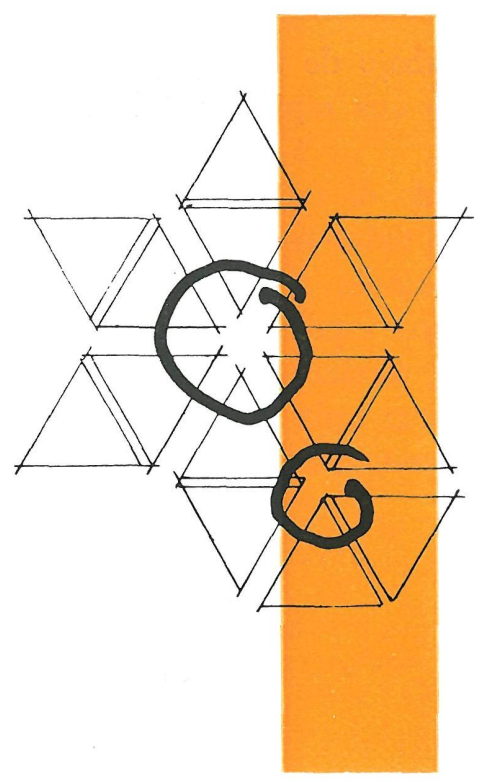

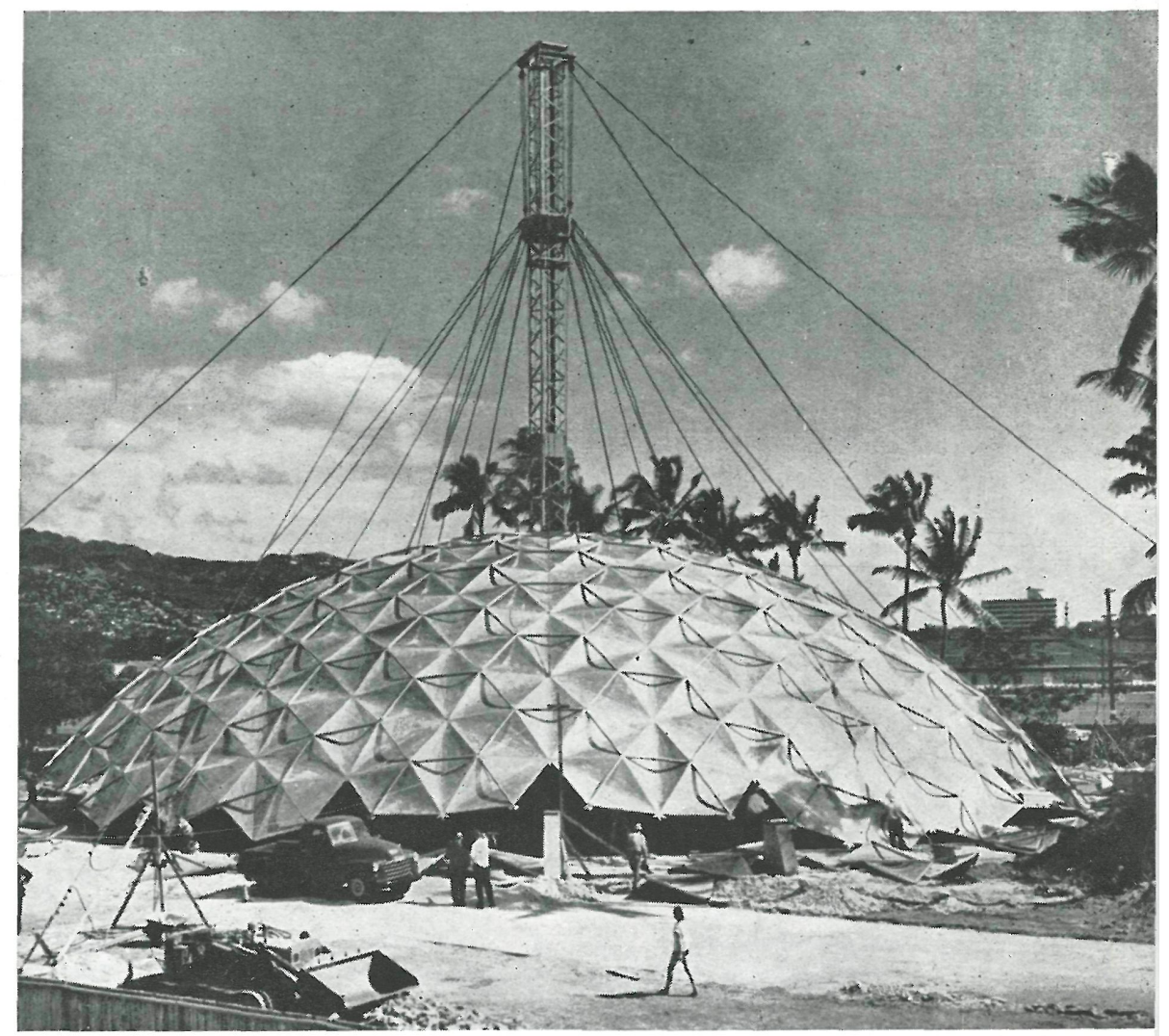

Por medio de un torno de mano se puede regular la altura que debe tener la cúpula en cada momento de su rápida construcción.

Montado este castillete central, se prepara un soporte provisional para apoyar el primer anillo de paneles.

Después se levanta este anillo con ayuda del torno y vientos, dejando su borde inferior a una altura del suelo tal, que permita montar, desde el suelo y sin andamios, el segundo anillo, procediendo así, sucesivamente, hasta terminar el último anillo.

Llegada esta fase, se procedió a la unión y anclaje de los puntos de apoyo del borde inferior con las placas de la parte superior de los soportes.

Cada panel va provisto de unas alas de unos $8 \mathrm{~cm}$ de anchura que, convenientemente perforados cada 15 centímetros en el sentido longitudinal, se van atornillando, sucesivamente, a medida que se van colocando los paneles. 


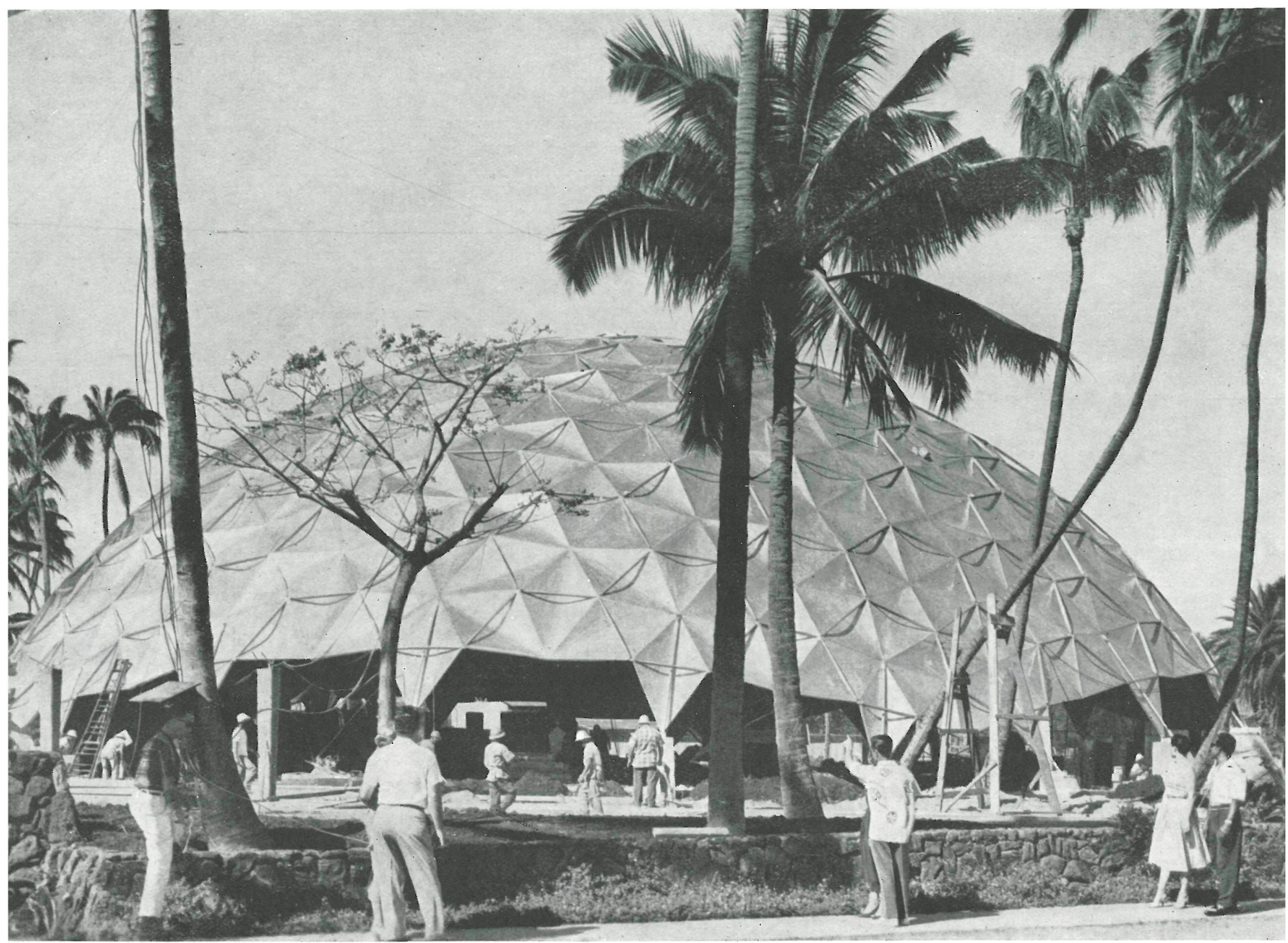

Fotos: KAISER ALUMINUM

Terminado el montaje, y después de anclar la cubierta, se retira el castillete central utilizado como medio auxiliar durante la construcción, y se procede a cerrar el hueco que dejó para dicho castillete.

Este pequeño hueco central, de perímetro pentagonal, se comporta como especie de ventosa para la ventilación, y con este objeto se le deja unos $10 \mathrm{~cm}$ por encima del borde de los paneles que la circundan.

La construcción de la cúpula propiamente dicha duró veinte horas de trabajo de un equipo de 38 operarios, lo cual supone una rapidez notable.

Para llegar a este grado de celeridad en la ejecución han de facilitarse todas las operaciones, reduciéndolas a manipulaciones sencillas coordinadas que no requieran una especialidad depurada. Un dato muy importante, tratándose de varios tipos de paneles, es el de marcar cada uno de éstos con un color claramente diferenciable.

J. J. U. 